\title{
Geometric Approach to Sampling and Communication
}

\author{
Emil Saucan \\ Department of Mathematics, Technion, Technion City \\ Haifa, 32000, Israel \\ semil@tx.technion.ac.il \\ Eli Appleboim \\ Electrical Engineering Department, Technion, Technion City \\ Haifa, 32000, Israel \\ eliap@ee.technion.ac.il \\ Yehoshua Y. Zeevi \\ Electrical Engineering Department, Technion, Technion City \\ Haifa, 32000, Israel \\ zeevi@ee.technion.ac.il
}

\begin{abstract}
Relationships that exist between the classical, Shannon-type, and geometric-based approaches to sampling are investigated. Some aspects of coding and communication through a Gaussian channel are considered. In particular, a constructive method to determine the quantizing dimension in Zador's theorem is provided. A geometric version of Shannon's Second Theorem is introduced. Applications to Pulse Code Modulation and Vector Quantization of Images are addressed.
\end{abstract}

Key words and phrases : Geometric sampling, Zador's Theorem, Vector quantization, Pulse code modulation, Lattice codes, Channel capacity, Shannon's Second Theorem.

2000 AMS Mathematics Subject Classification - Primary: 94A40, 94A20, 94A40; Secondary: 94A08, 57R05.

\section{General background}

\subsection{Introduction}

We consider a geometric approach to sampling, based on sampling the graph of the signal, considered as a manifold, rather than sampling in the domain of the signal. Whereas the latter is widely used in both theoretical and applied 
Signal and Image Processing, motivated by the framework of harmonic analysis, it is important to note that Shannon's original work is deeply rooted in the geometric approach, at least intuitively [51], [52]. Indeed, this geometric viewpoint of the problem distinguishes Shannon from Kotelnikov [25] and Nyquist [35], and allows one to transcend the restricted context of technical communication theory. We were also inspired in our endeavor by the "dictionary" of geometryto-communication-theory notions, and we strived to emulate it. Other paths towards the geometerization of Sampling Theory can also be found, e.g. in [37] and [24].

Having adopted a geometric approach to sampling, we are concerned with a broad spectrum of signals which can be categorized as geometric signals see Definition 1.3 below. In particular, we are concerned with images that are represented as multidimensional signals and embedded, as such, in $\mathbb{R}^{N}$, for some large enough $N$. This approach to signals and images has become very popular and well established in recent years, hence it calls for revisiting the basic issues of proper sampling of manifolds and related problems.

Our approach is based on the following sampling existence theorem for differentiable manifolds that was recently presented and applied in the context of Image Processing $([47],[48])^{1}$ :

Theorem 1.1 ([48]) Let $\Sigma$ be a connected, not necessarily compact smooth surface (i.e. of class $\mathcal{C}^{k}, k \geq 2$ ), with finitely many boundary components. Then, there exists a sampling scheme of $\Sigma$, with a metric density $\mathcal{D}=\mathcal{D}(p)=\mathcal{D}(\kappa)$, $\kappa=\max \left\{\omega_{M}, \frac{1}{k(p)}\right\}$, where $k(p)=\max \left\{\left|k_{1}\right|, \ldots,\left|k_{n}\right|\right\}$, and where $\omega_{M}$ is the maximal osculatory (or tubular) radius at $M,\left|k_{1}\right|, \ldots,\left|k_{n}\right|$ denoting the principal curvatures of $\Sigma$, at the point $p \in \Sigma$.

(For a more detailed exposition of the Differential Geometry and Topology notions, in particular for the definition of $\omega_{M}$, see the Appendix and [9]). That the density $\mathcal{D}$ is the correct and true extension of the notion of Nyquist rate of the classical sampling theory and algorithms, to "geometric signals", is shown in Section 5.1 of [48], where it is shown that, for the limiting case of 1-dimensional (i.e. classical) case the two notions, in fact, coincide. (While the term "metric" might be slightly redundant from the purely mathematical point of view, it is still needed to avoid confusion with the notion of density in Probability Theory.) Moreover, to the best of our knowledge, paper [48] and the present article represent the first comprehensive extension of the notion of Nyquist rate, as well as other Communication Theory notions to higher dimensional signals. Since, as already mentioned, the analogy of curvature rate with the Nyquist rate was discussed in detail in [48], we focus our attention in the present paper

\footnotetext{
${ }^{1}$ A similar approach to ours appeared in [29] but, mathematically less rigorous and comprehensive. Since we were not aware of this study upon the publication of our previous works [47], [1], we use this opportunity to rectify it.
} 
on exploring the geometric content of other Communication Theory notions. This endeavour represents the novel, original, contribution of this paper. It is addressed in Sections 2 and 3 below. (An exception is Section 2.3: The considerations therein appeared previously in [49], but are included here for the sake of completeness of the desired "dictionary" of geometry-to-communication-theory notions.) Some of the insights and formalism presented in this introductory section, are formulated here for the first time.

We should remark here that while for general manifolds, as well as for some applications such as in Graphics, the use of the tubular radius is essential, in the case of signals and images, it suffices to consider solely the principal curvatures. Moreover, as long as the analysis is local, there is no need to employ the computationally more difficult tubular radius (see the Appendix and, for more details, including illustrations, [48]).

It is important to note that Theorem 1.1 does not necessitate having exact locations of the sampling points. Thus, the above existence theorem lends itself to a stable constructive algorithm for sampling of manifolds and, therefore, of sampling of higher dimensional signals. Moreover, the following corollary is also applicable to this problem:

Corollary $1.2([48])$ Let $\Sigma^{n}, \mathcal{D}$ be as in Theorem 1.1. If there exists $k_{0}>0$, such that $k(p) \leq k_{0}$, for all $p \in \Sigma^{n}$, then there exists a sampling scheme of $\Sigma^{n}$ of finite density everywhere. In particular, if $\Sigma^{n}$ is compact, then there exists a sampling of $\Sigma^{n}$ having uniformly bounded density.

Note, however, that this is not necessarily the optimal scheme (see [48], [1]).

The constructive proof of this theorem (of which we outline in the Appendix just a sketch) is based on the existence of the so-called fat triangulations (see [39]). The density of the vertices of the triangulation (i.e. of the sampling) is given by the maximal principal curvature. An essential step in the construction of the triangulation consists of isometrically embedding of $\Sigma^{n}$ in some $\mathbb{R}^{N}$, for large enough $N$ (see [36]), where the existence of such an embedding is guaranteed by Nash's Theorem ([34]). Resorting to such a powerful tool as Nash's Embedding Theorem appears to be an impediment of our method, since the provided embedding dimension $N$ is excessively high (even after further refinements due to Gromov [17] and Günther [18]). Furthermore, even finding the precise embedding dimension (lower than the canonical $N$ ) is very difficult even for simple manifolds. However, as we shall indicate in the next section, this high embedding dimension actually becomes an advantage, at least from information theoretic viewpoint.

The resultant sampling scheme is in accord with the classical Shannon theorem, at least for the large class of (bandlimited) signals that also satisfy the condition of being $\mathcal{C}^{2}$ curves. In our proposed geometric approach, the radius of curvature substitutes for the condition of the Nyquist rate. To be more precise, 
our approach parallels, in a geometric setting, the local bandwidth of [19] and [62]. In other words, manifolds with bounded curvature represent a generalization of the locally band limited signals considered in those papers. However, the 1-dimensional case is a limiting, degenerated case, from the geometric viewpoint. As the notion of fatness (of simplices), essential to the geometric sampling scheme (see [48]), reduces, accounts for dihedral angles, and since on the real line we cannot impose such angles, the 1-dimensional version of Theorem 1.1 practically reduces to uniform sampling according to the Nyquist rate.

It should be stressed that, in comparing the classical and geometric approaches, one should bear in mind that no algebraic structure is presumed in the geometric context, whereas it is implicitly assumed in the (infinite) sum appearing in the classical version. Also, it should be noted that, in fact, Shannon already had the a deep geometric intuition (see, e.g. [52]) for phenomena that are studied traditionally (and certainly in Shannon's own time) using solely classical analytic tools. (How deep was Shannon's geometric intuition - inclusively for the correlation between curvature and entropy - was only recently revealed by the works of G. Perelman, regarding the Ricci flow, and C. Villani, on the Boltzman entropy.)

Here we further investigate the extent and implications of this analogy, and of the geometric approach in general. We begin by making, in the next Section, a few observations regarding the extent of our results, by finding the largest space of signals wherein our results may be applied effectively. Next, in Section 3, we establish the proper analogies considering concepts originating from classical sampling and from coding theory, considered in the context of Gaussian channels. In doing so, we attempt to construct a "dictionary" of geometric sampling and concepts originating from Shannon's fundamental approach [50].

As already mentioned, the paper concludes with an Appendix containing, for the sake of completeness and as a convenient reference, a brief review of the proof of Theorem 1.1.

\subsection{General geometric signals}

First, let us establish the following definition:

Definition 1.3 A geometric signal is the graph $\operatorname{Graph}(f)$ of a function $f$ : $\mathbb{R}^{m} \rightarrow \mathbb{R}^{n}$, appertaining to a desired class (e.g. $\left.L^{2}, \mathcal{C}^{2}, \mathcal{C}^{\infty}\right)$, endowed with a (natural) geometric structure.

The geometric structure, usually considered in vision and image processing, is that of a Riemannian manifold. This is also the approach we followed in proving Theorem 1.1, as emphasized in the previous section. However, the much larger class of Alexandrov spaces (see, e.g. [3]) can be considered in applications (see $[2])$. 
Example 1.4 The basic, motivating example of a geometric signal is that of gray scale images, viewed as surfaces $S$ in $\mathbb{R}^{3}, S=((x, y), h((x, y)))$, where $(x, y)$ represent the pixel coordinates and the function $h$ represents the gray scale level (intensity). In this case, the usual geometric structure considered is the Riemannian one induced by the ambient 3-dimensional Euclidean space. This is in particular the case when $S$ is considered to be a smooth surface. If, however, a Combinatorial (discrete) Image Processing viewpoint is adopted, then one can consider $S$ to be endowed only with its metric structure (inherited, again, from $\mathbb{R}^{3}$ ), see for instance, [45], [46].

As a followup of the definition and example above, unless stated otherwise, we consider signals as graphs of functions endowed with their natural geometric structure.

We begin our investigation by noting that, by the Paley-Wiener Theorem (see, e.g., [38]), any bandlimited signal is of class $\mathcal{C}^{\infty}$, and even analytic (perhaps under additional, but very mild, conditions). We have already shown in [47] that our geometric sampling method applies not only to bandlimited signals, but also to more general $L^{2}$ functions whose graphs are smooth $\mathcal{C}^{2}$ curves, not necessarily planar. In fact, the geometric sampling approach can be extended to a far larger class of manifolds. Indeed, every piecewise linear $(P L)$ manifold of dimension $n \leq 4$ admits a (unique, for $n \leq 3$ ) smoothing (see for example [55]), and every topological manifold of dimension $n \leq 3$ admits a $P L$ structure (cf., for example, [55]). In particular, for curves and surfaces, one can first consider a smoothing of class $\geq \mathcal{C}^{2}$ (so that curvature can be defined properly), which can then be sampled with sampling rate defined by the maximal curvature radius. Since a given manifold and its smoothing are arbitrarily close as smooth submanifolds of some $\mathbb{R}^{n}$ (i.e. in the fine $\mathcal{C}^{1}$ topology - see [33]), one obtains the desired sampling result. This very scheme is developed and applied in [47] for gray-scale images.

While numerical schemes for practical implementation of smoothing exist, they are not necessarily computationally satisfactory. For practical applications, one can circumvent this problem by applying numerical schemes based on the finite element method. However, for the sake of mathematical correctness, and in order to be able to tackle more general applications, one should consider more general curvature measures (see, e.g. [61]) and avoid smoothing altogether (see Section 2 below, for a brief discussion of this topic in a slightly different context).

It is worthwhile to highlight yet another aspect of our geometric sampling method: Shannon's Sampling theorem relates to bandlimited signals, that are, necessarily, unbounded in time or in spatial domain. Obviously, unbounded signals are not encountered in Signal/Image Processing, nor in any other practical implementations. However, geometric sampling does not necessitate having unbounded signals, quite the opposite: it is by far much easier to apply geometric sampling in cases of bounded manifolds. In practical implementations, this drastically reduces aliasing effects, while eliminating the need to produce periodic 
signals (surfaces).

\section{Important Implications}

\subsection{Pulse Code Modulation for Signals and Images}

Our geometrical approach to sampling lends itself to consideration of a broader range of topics in communications. In particular, it offers a new method for PCM (pulse code modulation) of images considered as high dimensional objects and not as a 1-dimensional signal obtained by some projection map (scan), of the image to a single dimension. The geometric approach is endowed with an inherent advantage in that the sampling points are associated with relevant geometric features (via curvature) and are not imposed arbitrarily at equal intervals determined by the Nyquist rate. To be more precise, each code word is represented, according to the proposed geometric approach, by a (sampling) point in some $\mathcal{R}^{N}$, belonging to the given geometric signal. The reconstruction of the signal is, basically, the piecewise flat one described in the Appendix. Of course, better results can be obtained if smoothing of the manifold is considered, especially if this is done using a curvature-based kernel [2]. This smoothing process requires one to encode also the curvature of the reconstructed piecewise linear manifold, and it is desirable to quantize curvature so that the number of extra bits added should be bounded. The specific number of bits is first of all a function of the geometry of the given signal/manifold, but also on several implementational/computational issues, that depend on the specific implementation and its restrictions (needed precision, desired computational speed, computational power, etc.) By the geometry of the manifold we refer first of all to the curvature itself (given that the sampling rate is based on this geometric invariant). Since in practice all signal/images are bounded, so will be (by a standard compactness argument) their curvature, or rather one should say, curvatures. Indeed, the choice of the curvature that one uses to sample the manifold is also a factor in determining the number of required bits. Indeed, if one uses the sampling method employed here, than the dimension of the manifold is another geometric invariant that one has to take into account, since one has to encode the $n$ principal curvatures $\left|k_{1}\right|, \ldots,\left|k_{n}\right|$ (where $n$ is the dimension of the manifold). On the other hand, if one chooses to sample the manifold using Ricci curvature, as proposed in [41], [43], one needs to encode just one additional, number, namely the Ricci curvature Ric (or, rather, its absolute value). The price to be payed for this apparent economy is multiple. The main loss is that of intuitiveness since, instead of working with an extrinsic notion (i.e. the principal curvatures) obtained from a concrete embedding in some $\mathbb{R}^{N}$, one uses an intrinsic notion (namely that of Ricci curvature) that is far less intuitive: While this is advantageous from the differential geometric viewpoint (as pointed out in [41]) it is 
far less useful for signal and image processing, for obvious reasons. Another problem with this approach resides in the faithfulness of the reconstruction (for a detailed discussion, both in the classical context, as well in that of "weighted" manifolds, see [43], [44]). Moreover, computing Ricci curvature requires, in the classical, smooth context, the computation of all sectional curvatures (see, e.g., [46] for a precise formula). On the other hand, approximating Ricci curvature for $P L$ manifolds is still a project that, except in dimension $n=2$, has no effective solution.

Moreover, it should be stressed again that the geometric approach, based on curvature radii, inherently produces a sparse, with respect to the Nyquist rate, adaptive sampling (see [1]), lending itself to interesting benefits, insofar as various applications are concerned. Indeed, the sampling density is lower in (almost) flat regions. Therefore, fewer sampling points are needed for such regions. (See [1] for more details.) A version of Landau's Theorem [26], [27] about non-uniform sampling is also attained and will be referred to again in Section 3. Moreover, given that the theoretical tubular radius (defined in Section 3 ), which is in general practically impossible to compute, is replaced by the curvature radii, and since for certain types of manifolds better global estimates may be obtained in terms of other curvature measures (e.g the Ricci curvature (see [1]), one can in some special cases, lower rates than the principal curvature rate given in Theorem 1.1.

\subsection{Vector Quantization for Images}

A complementary byproduct of the constructive proof of Theorem 1.1 is a precise method of vector quantization or block coding. Indeed, the proof of Theorem 1.1 consists of construction of a Voronoi (Dirichlet) cell complex $\left\{\bar{\gamma}_{k}^{n}\right\}$, whose vertices provide the sampling points. The set of centers $\left\{a_{k}\right\}$ of the cells, satisfying a certain geometric density condition, represent, as usual, the decision vectors. An advantage of this approach, besides its simplicity, is the possibility to estimate the error in terms of length and angle distortion, when reverting from the cell complex $\left\{\bar{\gamma}_{k}^{n}\right\}$ to the Euclidean cell complex $\left\{\bar{c}_{k}^{n}\right\}$ having the same set of vertices $\left\{\bar{\gamma}_{k}^{n}\right\}$ (see [36]).

\subsubsection{Error bounds}

Since the geometric method represented herein enables perfect reconstruction of the sampled manifold by an iterative process in which, at each iteration, a piecewise linear approximation is obtained it is important to have error bounds on the approximation at each iteration. It is shown in [47] (see appendix Theorem A3) that this error in the can be bounded in terms of the fatness coefficient of the obtained triangulation (defined in the appendix, Definition A.4) and of the diameter of simplices. 
Yet, for a more accurate error assessment, it is desirable to have bounds on the local distortion of the metric between the piecewise-flat approximation and the intrinsic metric of the manifold. Bounding this distortion of metrics bounds the distortion of geometries of the two objects (the approximation and the manifolds) say, in terms of curvature measures, volume diameter and other geometric attributes. This will allow us to say that the reconstructed approximation not only approximate the manifold pointwise, but also gives a good approximation to the geometry of the samples manifold. This is given by the following Theorem, [36]

Theorem 2.1 ([36]) If $M=M^{n}$ is a manifold without boundary, then locally, for any triangulation patch, the following inequality holds,

$$
\frac{3}{4} d_{M}(x, y) \leq d_{\text {eucl }}(\bar{x}, \bar{y}) \leq \frac{5}{3} d_{M}(x, y)
$$

where $d_{\text {eucl }}, d_{M}$ denote the Euclidean and intrinsic metric (on $M$ ) respectively, and where $x, y \in M$ and $\bar{x}, \bar{y}$ are their preimages on the piecewise-flat complex.

(The building of these patches is essential for the control of the fatness of the triangulation. Their size essentially depends upon the (local) maximal curvature - see [36] and the Appendix.)

For manifolds with boundary we have,

\section{Theorem $2.2([39])$}

$$
\frac{3}{4} d_{M}(x, y)-f(\theta) \eta_{\partial} \leq d_{e u c l}(\bar{x}, \bar{y}) \leq \frac{5}{3} d_{M}(x, y)+f(\theta) \eta_{\partial M}
$$

where $f(\theta)$ is a constant depending on the $\theta=\min \left\{\theta_{\partial M}, \theta_{\operatorname{int} M}\right\}$ - the fatness of the triangulation of $\partial M$ and int $\mathrm{M}$, respectively, and $\eta_{\partial}$ denotes the mesh of the triangulation (i.e. the supremum of the diameters of the simplices belonging to the triangulation) of a certain neighbourhood of $\partial M$ (see [39]).

In other words, the (local) projection mapping, $\pi$, between the triangulated manifold $M$ and its piecewise-flat approximation $\Sigma$ is (locally) bi-lipschitz if $M$ is open, but only a quasi-isometry (or coarsely bi-lipschitz) if the boundary of $M$ is not empty. (In fact, as the two inequalities above show, the projection mapping $\pi$ satisfies, in both cases, slightly stronger conditions.) Note that inequalities (1) and (2) imply that, both for open and bordered manifolds, control of the Euclidean error of the sampled points is equivalent to the control of the error for the sampled geometric signal. 


\subsection{Zador's Theorem}

Yet, a more important benefit of the proposed approach stems from Zador's Theorem [60]. ${ }^{2}$ The latter basically states that it is more efficient to use highdimensional quantizers [8]. This result implies that we can turn into an advantage the inherent curse of dimensionality. Indeed, by Zador's Theorem, the average mean-squared-error per dimension is:

$$
\mathcal{E}=\frac{1}{N} \int_{\mathbb{R}^{N}} d_{\text {eucl }}(x, p) p(x) d x,
$$

$p_{i}$ being the code-point closest to $x$, and $p(x)$ denotes the $p d f$ of $x$, can be obtained more efficiently by means of higher dimensional quantizers (see [8]).

Since for embedded manifolds, it obviously holds that $p(x)=p_{1}(x) \chi_{M}$, where $\chi_{M}$ is the characteristic function of $M$, defined on the ambient space $\mathbb{R}^{N}$, we obtain:

$$
\mathcal{E}=\frac{1}{N} \int_{M^{n}} d_{\text {eucl }}\left(x, p_{i}\right) p_{1}(x) d x .
$$

It follows that if the main issue is accuracy, and not simplicity, then 1-dimensional coding algorithms perform far worse than higher dimensional ones. Of course, there exists an upper limit for the coding dimension. Otherwise, one could just encode the whole data as one $N$-dimensional vector for $N$ large enough, which in practice may be huge. The geometric coding method proposed here provides a natural high dimension for the quantization of $M^{n}$ which is, the embedding dimension $N$. Moreover, it closes (at least for images and any other data that can be represented as a Riemannian manifold) the open problem (related to Zador's Theorem) of finding a constructive method to determine the dimension of a quantizer (Zador's proof is nonconstructive). In fact, for a uniformly distributed input (such as a manifold, and for example, as a noiseless image assumed to be), a better estimate of the average mean-squared-error per dimension can be obtained:

$$
\mathcal{E}=\frac{\frac{1}{N} \int_{M^{n}} d_{\text {eucl }}\left(x, p_{i}\right) d x}{\int_{M^{n}} d x}=\frac{\frac{1}{N} \int_{M^{n}} d_{e u c l}\left(x, p_{i}\right) d x}{\mathcal{V}_{n}\left(M^{n}\right) d x},
$$

where $\mathcal{V}_{n}$ denotes the $n$-dimensional volume (area) of $M$. Whence, for compact manifolds, one obtains the following expression for $\mathcal{E}$ :

$$
\mathcal{E}=\frac{\frac{1}{N} \int_{M^{n}} d_{\text {eucl }}\left(x, p_{i}\right) d x}{\sum_{i}^{m} \int_{V_{i}} d x}=\frac{\frac{1}{N} \int_{M^{n}} d_{\text {eucl }}\left(x, p_{i}\right) d x}{\sum_{i}^{m} \mathcal{V}_{n}\left(V_{i}\right) d x},
$$

\footnotetext{
${ }^{2}$ Zador's paper represents a revised version of a much earlier and practically obscure Technical Report [59], itself based upon Zador's PhD Thesis [58]. As already mentioned, Zador's work was largely unknown till the publication of Gersho's paper [14]. (For an excellent, exhaustive presentation of the history and the multifaceted aspects of this theorem and various other aspects of Quantization Theory, see [16].)
} 
where $\left\{V_{i}\right\}$ represent the Voronoi cells of the partition. Moreover, we have the following estimate for the quantizer: Choose centers of cells such that the quantity

$$
\mathcal{Q}=\frac{1}{N} \frac{\frac{1}{m} \int_{M^{n}} d_{\text {eucl }}\left(x, p_{i}\right) d x}{\left(\frac{1}{m} \sum_{i}^{m} \mathcal{V}_{n}\right)^{1+\frac{2}{N}}}
$$

is minimized.

The embedding dimension $N$ increases dramatically, even for compact manifolds and even taking into consideration Gromov's and Günther's improvements of Nash's original method (see [17], resp. [18]). For instance, $n=2$ requires embedding dimension $N=10$ and $n=3$ the necessitates $N=14$. Hence, for large enough $n$ one can write following rough estimate:

$$
\mathcal{Q} \approx \frac{1}{N} \frac{\int_{M^{n}} d_{e u c l}\left(x, p_{i}\right) d x}{\sum_{i}^{m} \mathcal{V}_{n}}
$$

\section{Sampling and Codes}

\subsection{Packings, Coverings and Lattice codes}

According to classical signal processing theory, the required signal bandwidth $W$ and the Nyquist sampling rate are constrained by the condition $W=\eta / 2$, where $W$ and $\eta$ are the bandwidth and sampling rate respectively. This lends itself to an immediate generalization to periodic signals, or, in geometric terms, for signals represented over a lattice: $\Lambda=\left\{\lambda_{i}\right\}$. One can even interpret the boundaries of the lattice as the coordinates in a multi-dimensional (warped) space or time (see, e.g. [54], [31]). (Alternatively, one can interpret the dimensions as representing wave-length, or even as combined fundamental quantities, e.g. spacetime or even space-time-wave-length, as they arise in Medical Imaging (CT).) Note that such signals can be viewed as distributions on the $n$-dimensional torus $\mathbb{T}^{n}=\mathbb{R}^{n} / \mathbb{Z}^{n}$. According to this interpretation, the ( $n$-dimensional!) period is the fundamental cell $\lambda$ of the lattice. Two scalars are naturally associated with this cell: its diameter, $\operatorname{diam}(\lambda)$ (or, alternatively, the length of the longest edge), and its volume $\operatorname{Vol}(\lambda)$. Either one may be used as a measure of the $n$-dimensional period. However, they are both interrelated and associated with one geometric feature, the so-called "fatness":

Definition 3.1 Let $\gamma=\gamma^{k}$ be a k-dimensional cell. The fatness (or aspectratio) of $\gamma$ is defined as:

$$
\varphi(\gamma)=\min _{\lambda} \frac{\operatorname{Vol}(\lambda)}{\operatorname{diam}^{l}(\lambda)}
$$


where the minimum is taken over all the $l$-dimensional faces of $\gamma, 0 \leq k$. (If $\operatorname{dim} \lambda=0$, then $\operatorname{Vol}(\lambda)=1$, by convention.)

In the case of simplices (and, since any cell can be canonically decomposed into simplices, of regular cells) this definition of fatness is equivalent to the following one (see [36]):

Definition $3.2 A k$-dimensional cell $\gamma$ is called $\varphi$-fat if there exists $\varphi>0$ such that the ratio $\frac{r}{R} \geq \varphi$; where $r$ denotes the radius of the inscribed sphere of $\gamma$ (or in-radius) and $R$ denotes the radius of the circumscribed sphere of $\gamma$ (or circum-radius). A cell-complex $\Gamma=\left\{\gamma_{i}\right\}_{i \in \mathbf{I}}$ is fat if there exists $\varphi \geq 0$ such that all its cells are $\varphi$-fat.

Recall that the in- and circum-radius are important in lattice problems: given a lattice $\Lambda$ with (dual) Voronoi cell $\Pi$ (of volume 1), one has to minimize the in-radius, to solve the packing problem, and to minimize the circum-radius for solving the covering problem (see [8]). Note that $\Lambda$ and $\Pi$ are simultaneously fat. It follows that fat cell-complexes and, in particular, fat triangulations, represent a mini-max optimization for both the packing and the covering problem. Moreover, since fat triangulations are essential for the sampling theorem for manifolds, it appears that there exists an intrinsic relation between the sampling problem for manifolds and the covering and packing problems.

\subsection{Average Power, Rate of Code and Channel Capacity}

It is natural to extend the classical definitions of average power of a signal:

$$
P=\frac{1}{T} \int_{0}^{T} f^{2}(t) d t
$$

and the rate of a code:

$$
R=\frac{1}{T} \log _{2} N,
$$

in the context of lattices with fundamental cell $\lambda$, where $N$ represents the number of code points, in the following manner:

$$
P=\frac{1}{\operatorname{Vol}(\Lambda)} \int_{\lambda} f^{2}(t) d t=\frac{1}{N_{1} \operatorname{Vol}(\lambda)} \int_{\lambda} f^{2}(t) d t
$$

and

$$
R=\frac{1}{\operatorname{Vol}(\Lambda)} \log _{2} N=\frac{1}{N_{1} \operatorname{Vol}(\lambda)} \log _{2} N,
$$

respectively, $N_{1}$ being the number of cells. 
Similarly, one can adapt the classical definition of the channel capacity:

$$
C=\lim _{T \rightarrow \infty} R=\lim _{T \rightarrow \infty} \frac{\log _{2} N}{T},
$$

to become

$$
C=\lim _{T \rightarrow \infty} \frac{\log _{2} N}{\operatorname{Vol}(\Lambda)}=\lim _{T \rightarrow \infty} \frac{1}{N_{1} \operatorname{Vol}(\lambda)} \log _{2} N .
$$

Since $N$ and $N_{1}$ are related by $N_{1}=\alpha(N)$, where $\alpha$ is the growth function of the manifold, the expression of channel capacity, $C$, becomes:

$$
C=\lim _{T \rightarrow \infty} \frac{1}{\operatorname{Vol}(\lambda)} \frac{\log _{2} N}{\alpha(N)} .
$$

It follows that $C=\infty$ for non-compact Euclidean and Hyperbolic manifolds, and $C=0$ for their Elliptic counterparts. Unfortunately, no such immediate estimates can be easily produced for manifolds of variable curvature.

Note that by substituting $1 / T=\operatorname{Vol}(M)$, the above definitions apply to any sampling scheme of any manifold of finite volume, not just to lattices. In this case $N$ and $N_{1}$ represent the number of vertices, respective simplices, of the triangulation. In the context of classical signal processing, this approach is known as "recurrent nonuniform sampling" [11]. (See also [57].)

The interpretation of frequency considered above does not extend, however, to general geometric signals. For a proper generalization we have to look into the geometric analogue of $W$. Based on [48], according to Theorem 5.2, for the case of curves, i.e 1-dimensional (geometric) signals, $W$ equals the curvature rate $k / 2$, were $k$ represents the maximal absolute curvature of the curve. This, and the sampling Theorem 4.11 of [48], naturally lend themselves to the following definition of $W$ for general geometric signals:

Definition 3.3 Let $M=M^{n}$ be an n-dimensional manifold $n \geq 2$. $W=W_{M}=$ $1 / k_{M}$, where $k_{M}=\max k_{i}$ and $k_{i}, i=1, \ldots, n$ are the principal curvatures of $M$.

According to classical considerations, the energy $E$ of the signal $f: \mathbb{R} \rightarrow \mathbb{R}$ is considered to be equal to its $L^{2}$ norm:

$$
E=E(f)=\int_{-\infty}^{\infty} f^{2}(t) d t=\frac{1}{2 W} \sum_{k=-\infty}^{+\infty} f^{2}\left(\frac{k}{2 W}\right) .
$$

One would like, of course, to find proper generalizations of the notion of energy for more general (geometric) signals. In view of the above discussion, the first step is to replace $2 W$ by the proper generalized expression. However, when considering more general function spaces of specific relevance (s.a. bounded variation $(B V)$, bounded oscillation $(B O)$, bounded mean oscillation $(B M O))$, one 
should consider energies fitting the specific norm of the space under consideration. This discussion is, of course, also valid with regard to the average power $P$, and rate $R$, of a geometric signal.

We proceed to consider the first definition of code efficiency: the nominal coding gain (ncg) of a code $c_{1}$ over another code, say $\left(c_{2}\right)$, is defined as:

$$
\operatorname{ncg}(C 1, C 2)=10 \log _{10}\left(\frac{\mu_{1}}{E_{1}} / \frac{\mu_{2}}{E_{2}}\right),
$$

where $\mu$ is the square of the minimal squared-distance between coding points. For geometric codes of bounded curvature (hence compact codes), the expression for $\mu$ is in particular simple: $\mu=1 / \min k$ ( $k$ denoting again principal curvature).

\subsection{The Channel Coding Problem}

It is most natural to approach problems associated with the Gaussian white noise channel in the context of "geometric signals", i.e. in the context of manifolds. Recall that in the classical context, a received signal is represented by a vector $X=F+Y$, where $F=\left(f_{1}, \ldots, f_{N}\right)$ is the transmitted signal, and $Y=\left(y_{1}, \ldots, y_{n}\right)$ represents the noise, whose components $y_{i}$ are independent Gaussian random variables, of mean 0 and average power $\sigma^{2}$. The main, classical result for the Gaussian channel is the following:

Theorem 3.4 (Shannon's Second Theorem, [51]) For any rate $R$ not exceeding the channel capacity $C_{0}$,

$$
C_{0}=\frac{1}{T} \log _{2}\left(1+\frac{P}{\sigma^{2}}\right),
$$

there exists a sufficiently large $T$, such that there exists a code of rate $R$ and average power $\leq P$, such that the probability of a decoding error is arbitrarily small. Conversely, it is not possible to obtain arbitrarily small errors for rates $R>C_{0}$.

In the case of geometric signals, the transmitted signal $F$ is given by its sampling (code) points on the corresponding manifolds. Since the mean equals 0 , the noisy transmitted signal $F+Y$ lies in the tube $\operatorname{Tub}_{\sigma}(M)$, where tubes are defined as follows:

Definition 3.5 Let $M \subset \mathbb{R}^{m}$ be an orientable embedded manifold, and let $\bar{N}_{p}$ denote the unit normal to $M$ at the point $p$. For each $p \in M$ consider the open symmetric interval of length $2 \varepsilon_{p}, I_{p, \varepsilon_{p}}$, in the direction of $\bar{N}_{p}$, where $\varepsilon_{p}$ is to be chosen small enough such that $I_{p, \varepsilon_{p}} \cap I_{q, \varepsilon_{q}}=\emptyset$, for all $p, q \in M,\|p-q\|_{2}>$ $\xi \in \mathbb{R}_{+}$. Then $\operatorname{Tub}_{\varepsilon}(M)=\bigcup_{p \in M} I_{p}$ is an open set that contains $M$, such that for any point $x \in T u b_{\varepsilon}(M)$ there exists a unique line normal to $M$ that passes through $x . T_{u}(M)$ is called a tubular neigbourhood of $M$ or just a tube. 
The existence of tubular neighborhoods is assured both locally, for any regular, orientable manifold, and globally for regular, compact, orientable manifold (see [15]). In addition, the regularity of the manifolds $\partial \operatorname{Tub}_{\sigma}^{-}(M)=$ $M-\bigcup_{p \in M} \varepsilon \bar{N}_{p}, \partial \operatorname{Tub}_{\sigma}^{+}(M)=M+\bigcup_{p \in M} \varepsilon \bar{N}_{p}$, where $\partial \operatorname{Tub}_{\sigma}^{-}(M) \cup \partial \operatorname{Tub}_{\sigma}^{+}(M)=$ $\partial \operatorname{Tub}_{\sigma}(M)$, is at least as high as that of $M$ : If $M$ is convex, then $\partial \operatorname{Tub}_{\sigma}^{-}(M)$, $\partial \operatorname{Tub}_{\sigma}^{+}(M)$ are piecewise $\mathcal{C}^{1,1}$ manifolds (i.e. they admit parameterizations with continuous and bounded derivatives), for all $\sigma>0$. Also, if $M$ is a smooth enough manifold with a boundary, that is, at least piecewise $\mathcal{C}^{2}$, then $\partial \operatorname{Tub}_{\sigma}^{-}(M)$, $\partial \operatorname{Tub}_{\sigma}^{+}(M)$ are piecewise $\mathcal{C}^{2}$ manifolds, for all small enough $\sigma$ (see [12]).

In the geometric setting, $\sigma$ can be taken, of course, to be the maximal Euclidean deviation. However, a better deviation measure is, at least for compact manifolds, the Haussdorf Distance (between $M$ and $\partial \operatorname{Tub}_{\sigma}^{-}(M), \partial \operatorname{Tub}_{\sigma}^{+}(M)$ ), defined as follows:

Definition 3.6 Let $(X, d)$ be a metric space and let $A, B \subseteq(X, d)$. The Hausdorff distance between $A$ and $B$ is defined as:

$$
d_{H}(A, B)=\max \left\{\sup _{a \in A} d(a, B), \sup _{b \in B} d(b, A)\right\}
$$

For non-compact manifolds, one has to consider the more general GromovHausdorff distance (see, for example, [3]).

Since, according to the above arguments, both the distance between $M$ and $\partial \operatorname{Tub}_{\sigma}^{-}(M), \partial \operatorname{Tub}_{\sigma}^{+}(M)$ and the deviations of their curvature measures are arbitrarily small for small enough $\sigma$, we can state a first qualitative geometric version of Shannon's Theorem for the Gaussian channel. While a perfect analogy is not available, we can nevertheless formulate the following theorem:

Theorem 3.7 Let $M^{n}$ be a smooth geometric signal (manifold) and let $\sigma$ be small enough, such that $\operatorname{Tub}_{\sigma}(M)$ is a submanifold of $\mathbb{R}^{n+1}$. Then, given any noisy signal $M+Y$, such that the average noise power $\sigma_{Y}$ is at most $\sigma$, there exists a sampling scheme of $M+Y$ with an arbitrarily small probability of resultant decoding error.

The analogue of the capacity in the context of the geometric approach to codes is $C_{0}=C_{0}(n, \sigma, r)$, where $r$ represents the differentiability class of $M$.

The existence of tube $\partial \operatorname{Tub}_{\sigma}^{+}(M)$ is, as noted, guaranteed globally in the case of compact manifolds. Hence it follows that the sampling scheme is also global and necessitates $O(N)$ points, $N=N_{M}$. However, for non-compact manifolds (in particular non-band limited geometric signals), the existence of $\partial \operatorname{Tub}_{\sigma}^{+}(M)$ is guaranteed only locally. Therefore "gluing "of patches is needed, an operation which requires the insertion of additional vertices (i.e. sampling points), their number being a function of the dimension of $M$. Hence, in this case, $N_{M+Y}=O\left(N_{M}^{n}\right)$. 
It is important to note that, again, this result is not restricted to smooth manifolds, but rather extends to much more general signals: Indeed, for any compact set $M \in \mathbb{R}^{n}$, the $(n-1)$-dimensional sets $\partial \operatorname{Tub}_{\sigma}^{-}(M), \partial \operatorname{Tub}_{\sigma}^{+}(M)$, are Lipschitz manifolds(i.e. topological manifolds equipped with a maximal atlas for which the changes of coordinates are Lipschitz functions), for almost any $\varepsilon$ (see $[20])$. Moreover, the generalized curvatures measures of $\partial \operatorname{Tub}_{\sigma}^{-}(M), \partial \operatorname{Tub}_{\sigma}^{+}(M)$ are arbitrarily close to the curvature of $M$, for small enough $\sigma$ ([7], [20]). It follows that the above generalization befits not only the case of the Gaussian noise, but to more general types of noise, as well (see, [51], [21], [28]).

We note also en passant, that practically the same argument as above, with little (if any) modifications allows us to obtain a geometric version of Landau's result on the reconstruction of distorted signals, [32].

The full details of a quantitative version, including the general case, are however, more involved and warrant a more detailed consideration elsewhere.

Before we conclude this section, we wish to emphasize that the importance of tubes is not necessarily limited to Differential Geometry. It is just as important in Statistics (see, e.g. [30]). However, its relevance to sampling theory is in particular evident. Indeed, Shannon's ideas, as exposed in [50] and [51] are very similar to our approach (even if lacking the specific geometric nomenclature). In particular, convergence of the measure of entropy of the noise introduced in [51] is easily obtained in the context of our approach and formalism. For instance, the equivalence of the tube radius is used in [50] as a measure of the uncertainty of the reconstruction (not to be confused with the Heisenberg-related Uncertainty Principle). For the development of Shannon's approach see [53].

\section{ACKNOWLEDGEMENT}

First author's research supported by European Research Council under the European Community's Seventh Framework Programme (FP7/2007-2013) / ERC grant agreement $\mathrm{n}^{\circ}$ [203134]. He would also like to thank Professors Shahar Mendelson and Gershon Wolansky - their warm support is gratefully acknowledged. We thank Professor Shlomo Shamai for pointing out that the geometric sampling approach actually motivated Shannon, and that this approach is inherent in his pioneering work. This Research has been supported in part by the Ollendorf Minerva Center and by a special grant awarded by the Minerva Foundation to Y. Y. Zeevi for investigation of signal and image representations.

\section{References}

[1] E. Appleboim, E. Saucan and Y.Y. Zeevi, Geometric Sampling for Signals with Applications to Images, Proceedings of SampTA '07, 1-6, 2008. 
[2] E. Appleboim, E. Saucan and Y.Y. Zeevi, Geometric Reproducing Kernels for Signal Reconstruction, Proceedings of SampTA '09, http://hal.archivesouvertes.fr/docs/00/49/54/56/PDF /

SampTAProceedings.pdf, 291-294.

[3] D. Burago, Y. Burago, and S. Ivanov, Course in Metric Geometry, GSM 33, AMS, Providence, 2000.

[4] S.S. Cairns, A simple triangulation method for smooth manifolds, Bull. Amer. Math. Soc., 67, 1961, 389-390.

[5] E.J. Candès, Compressive sampling, Proceedings of the International Congress of Mathematicians, Madrid, Spain, 2006.

[6] J. Cheeger, W. Müller and R. Schrader, On the Curvature of Piecewise Flat Spaces, Comm. Math. Phys., 92, 405-454, 1984.

[7] J. Cheeger, W. Müller and R. Schrader, R. Kinematic and Tube Formulas for Piecewise Linear Spaces, Indiana Univ. Math. J., 35 (4), 737-754, 1986.

[8] J.H. Conway and N.J.A. Sloane, Sphere Packings, Lattices and Groups, third ed., Springer, New York, 1999.

[9] M.P. do Carmo, Differential Geometry of Curves and Surfaces, PrenticeHall, Englewood Cliffs, N.J., 1976.

[10] D.L. Donoho and C. Grimes, Hessian eigenmaps: Locally linear embedding techniques for high-dimensional data, PNAS, 100(10), 5591-5596, 2003.

[11] Y.C. Eldar and A.V. Oppenheim, Filter Bank Reconstruction of Bandlimited Signals From Nonuniform and Generalized Samples, IEEE Trans. Signal Processing, 48, 2864-2875, 2000.

[12] H. Federer, Curvature measures, Trans. Amer. Math. Soc., 93, 418-491, 1959.

[13] D. Gabor, Theory of communication, J. Inst. Elect. Eng. (London), 93, pt. 3(26), 429, 1946.

[14] A. Gersho, Asymptotically optimal block quantization, IEEE Trans. Inform. Theory, vol. IT-25, 373?380, July 1979

[15] Gray, A. Tubes, Addison-Wesley, Redwood City, Ca, 1990.

[16] R.M. Gray and D.L. Neuhoff, Quantization, IEEE Transactions on Information Theory, 44(6), 2325-2383, 1998. 
[17] M. Gromov, M. Partial differential relations, Springer-Verlag, Ergeb. der Math. 3 Folge, Bd. 9, Berlin - Heidelberg - New-York, 1986.

[18] M. Günther, Isometric embeddings of Riemannian manifolds, Proc. ICM Kyoto, 1137-1143, 1990.

[19] K. Horiuchi, Sampling principle for continuous signals with time-varying bands, Information and Control, 13(1), 53?61, 1968.

[20] J. Howland and J.H.G. Fu, Tubular neighbourhoods in Euclidean spaces, Duke Math. J., 52(4), 1025-1045, 1985.

[21] J. Huang, Statistics of Natural Images and Models, PhD Thesis, Brown University, 2000.

[22] A.K. Jain and W.K. Pratt, Color Image Quantization, IEEE Publication 72 CHO 601-5-NTC, National Telecommunication Conference 1972 Record, Huston, TX, 1972.

[23] D.B. Judd, D.L. MacAdam and G Wyszecki, Spectral Distribution of typicl daylight as a function of correlated color temperature, J. Opt. Soc. Am., 53, 1031-1040, 1964.

[24] A. Kempf and R. Martin Towards sampling theory on curved manifold, Proceedings of SampTA 07, 2008.

[25] V.A. Kotelnikov, On the transmission capacity of the'ether' and of cables in electrical communications, (Bissel, C. C. and Katsenelson, V.A., transl.), Proc. of the first All-Union Conference on the technological reconstruction of the communications sector and low-current engineering, Moscow, 1933.

[26] H.J. Landau, Necessary Density Conditions for Sampling and Interpolation of Certain Entire Functions, Acta Math., 117, 37?52, 1967.

[27] H.J. Landau, Sampling, Data Transmission, and the Nyquist Rate, Proc. IEEE, 55, 1701-1706, 1967.

[28] A.B. Lee, K.S. Pedersen D. and Mumford, The Nonlinear Statistics of HighContrast patches in Natural Images, Int. J. Comput. Vis., 54(1/2/3), 831003, 2003.

[29] G. Leibon and D. Letscher, Delaunay Triangulations and Voronoi Diagrams for Riemannian Manifolds, Proceedings of the Sixteenth Annual Symposium on Computational Geometry, 341-349, 2000.

[30] C. Loader, The Volume-of-Tubes Formula: Computational Methods and Statistical Applications, preprint, arXiv:math/0511502v1, 2005. 
[31] A.H. Louie, Multidimensional time: A much delayed chapter in a phenomenological calculus, preprint, 2004.

[32] W.L. Miranker and H.J. Landau, The Recovery of Distorted Band-Limited Signals, J. Math. Anal. and Appl., 2(1), 97?104, 1961.

[33] J.R. Munkres, Elementary Differential Topology, (rev. ed.) Princeton University Press, Princeton, N.J., 1966.

[34] J. Nash, The embedding problem for Riemannian manifolds, Ann. of Math. (2), 63, 20-63, 1956.

[35] H. Nyquist, Certain topics in telegraph transmission theory, Trans. AIEE, 47, 363-390, 1928.

[36] K. Peltonen, On the existence of quasiregular mappings, Ann. Acad. Sci. Fenn., Series I Math., Dissertationes, 1992.

[37] I. Pesenson, A sampling theorem on homeogeneous manifolds, Trans. Amer. Math. Soc., 352(9), 4257-4269, 2000.

[38] J. Ramanathan, Methods of Applied Fourier Analysis, Birkhäuser, Boston, Ma., 1998.

[39] E. Saucan, Note on a theorem of Munkres, Mediterr. j. math., 2(2), 2005, $215-229$.

[40] E. Saucan, Curvature - Smooth, Piecewise-Linear and Metric, in What is Geometry (G. Sica, editor), Advanced Studies in Mathematics and Logic, 237-268, Polimetrica, Milano, 2006.

[41] E. Saucan, Intrinsic Differential Geometry and the Existence of Quasimeromorphic Mappings, Revue Roumaine de Math. Pures et Appl., 54(5-6), 565$574,2009$.

[42] E. Saucan, Geometric Sampling of Infinite Dimensional Signals, Sampl. Theory Signal Image Process., 10(1-2), 59-76, 2011.

[43] E. Saucan, Curvature based triangulation of metric measure spaces, Contemporary Mathematics, 554, 207-227, 2011.

[44] E. Saucan, A Simple Sampling Method for Metric Measure Spaces, preprint, arXiv:1103.3843v1 [cs.IT], 2011.

[45] E. Saucan and E. Appleboim, Metric Methods in Surface Triangulation, Lecture Notes in Computer Science, 5654, 335?-355, 2009. 
[46] E. Saucan, E. Appleboim, G. Wolansky and Y.Y. Zeevi, Combinatorial Ricci Curvature and Laplacians for Image Processing, Proceedings of CISP'09, Vol. 2, 992-997, 2009.

[47] E. Saucan, E. Appleboim and Y.Y. Zeevi, Geometric Sampling of Manifolds for Image Representation and Processing, SSVM 2007, Lecture Notes in Computer Science, 4485, 907-918, Springer-Verlag, 2007.

[48] E. Saucan, E. Appleboim and Y.Y. Zeevi, Sampling and Reconstruction of Surfaces and Higher Dimensional Manifolds, J. Math. Imaging. Vis., 30(1), 105-123, 2008.

[49] E. Saucan, E. Appleboim and Y.Y. Zeevi, Geometric Sampling of Images, Vector Quantization and Zador's Theorem, Proceedings of SampTA 2009, http://hal.archives-ouvertes.fr/docs/00/49/54/56/PDF/

SampTAProceedings.pdf, 267-269.

[50] C.E. Shannon, A Mathematical Theory of Communication, Bell System Technical Journal, 27, 379-423, 623-656, 1948.

[51] C.E. Shannon, Communication in the presence of noise, Proceedings of the IRE, 37(1), 10-21, 1949.

[52] C.E. Shannon, Some Geometrical Results in Channel Capacity, Nachrichtentechnische Zeit, 10, 259-264, 1957.

[53] S. Smale and D.X. Zhou, Shannon sampling and function reconstruction from point values, Bull. Amer. Math. Soc., 41(3), 279-305, 2004.

[54] J. Strnad, On multidimensional time, J. Phys. A: Math. Gen., 13, 389-391, 1980.

[55] W. Thurston, Three-Dimensional Geometry and Topology, vol.1, (Edited by S. Levy), Princeton University Press, Princeton, N.J. 1997.

[56] M. Unser, Sampling - 50 Years After Shannon, Proceedings of the IEEE, 88(4), 569-587, 2000.

[57] D. Walnut, Nonperiodic Sampling of bandlimited Functions on Unions of Rectangular Lattices, J. Fourier. Anal. Appl., 2(5), 435-452, 1996.

[58] P.L. Zador, Development and evaluation of procedures for quantizing multivariate distributions, Ph.D. dissertation, Stanford Univ., 1963, also Stanford Univ. Dept. Statist. Tech. Rep.

[59] P.L. Zador, Topics in the asymptotic quantization of continuous random variables, Bell Lab. Tech. Memo., 1966. 
[60] P.L. Zador, Asymptotic Quantization Error of Continuous Signals and the Quantization Dimension, IEEE Trans. on Info. Theory, 28(22), 139-149, 1982. (revised version of [59].)

[61] M. Zähle, Curvature Theory for Singular Sets in Euclidean Spaces, preprint.

[62] Y.Y. Zeevi and E. Shlomot, Nonuniform sampling and antialiasing in image representation, IEEE Trans. Signal Process., 41(3), 1223-1236, 1993.

\section{Appendix - A Concise Proof of Theorem 1.1}

The proof of Theorem 1.1 is essentially based on existence of fat triangulation for Riemannian manifolds. In the sequel we outline some definitions and notations, and review relevant results concerning the existence of such triangulations.

Let $M^{n}$ denote an $n$-dimensional complete Riemannian manifold, and let it be isometrically embedded into $\mathbb{R}^{N}$ ("N"-s existence is guaranteed by Nash's Theorem - see, e.g. [36]).

Let $\mathbb{B}^{\nu}(x, r)=\left\{y \in \mathbb{R}^{\nu} \mid d_{\text {eucl }}(x, y)<r\right\} ; \partial \mathbb{B}^{\nu}(x, r)=\mathbb{S}^{\nu-1}(x, r)$. If $x \in M^{n}$, let $\sigma^{n}(x, r)=M^{n} \cap \mathbb{B}^{\nu}(x, r), \beta^{n}(x, r)=\exp _{x}\left(\mathbb{B}^{n}(0, r)\right)$, where: $\exp _{x}$ denotes the exponential map: $\exp _{x}: T_{x}\left(M^{n}\right) \rightarrow M^{n}$ and where $\mathbb{B}^{n}(0, r) \subset T_{x}\left(M^{n}\right)$, $\mathbb{B}^{n}(0, r)=\left\{y \in \mathbb{R}^{n} \mid d_{\text {eucl }}(y, 0)<r\right\}$.

The following definitions generalize in a straightforward manner classical ones used for surfaces in $\mathbb{R}^{3}$ :

Definition A.1 1. $\mathbb{S}^{\nu-1}(x, r)$ is tangent to $M^{n}$ at $x \in M^{n}$ iff there exists $\mathbb{S}^{n}(x, r) \subset \mathbb{S}^{\nu-1}(x, r)$, s.t. $T_{x}\left(\mathbb{S}^{n}(x, r)\right) \equiv T_{x}\left(M^{n}\right)$.

2. Let $l \subset \mathbb{R}^{\nu}$ be a line, then $l$ is secant to $X \subset M^{n}$ iff $|l \cap X| \geq 2$.

Definition A.2 1. $\mathbb{S}^{\nu-1}(x, \rho)$ is an osculatory sphere at $x \in M^{n}$ iff:

(a) $\mathbb{S}^{\nu-1}(x, \rho)$ is tangent at $x$; and

(b) $\mathbb{B}^{n}(x, \rho) \cap M^{n}=\emptyset$.

2. Let $X \subset M^{n}$. The number $\omega=\omega_{X}=\sup \left\{\rho>0 \mid \mathbb{S}^{\nu-1}(x, \rho)\right.$ osculatory at any $x \in X\}$ is called the maximal osculatory (tubular) radius at $X$.

Remark A.3 There exists an osculatory sphere at any point of $M^{n}$ (see [4]).

\section{A1 Fat triangulations}

Definition A.4 1. A triangle in $\mathbb{R}^{2}$ is called $\varphi$-fat iff all its angles are larger than a prescribed value $\varphi>0$. 
2. A $k$-simplex $\tau \subset \mathbb{R}^{n}, 2 \leq k \leq n$, is $\varphi$-fat if there exists $\varphi>0$ such that the ratio $\frac{r}{R} \geq \varphi$, where $r$ and $R$, are respectively, the radii of the inscribed and circumscribed ( $k-1)$-spheres of $\tau$.

3. A triangulation $\mathcal{T}=\left\{\sigma_{i}\right\}_{i \in \mathbf{I}}$ is fat if all its simplices are $\varphi$-fat for some $\varphi>0$.

Proposition A.5 ([6]) There exists a constant $c(k)$ that depends solely upon the dimension $k$ of $\tau$ such that

$$
\frac{1}{c(k)} \cdot \varphi(\tau) \leq \min _{\sigma<\tau} \measuredangle(\tau, \sigma) \leq c(k) \cdot \varphi(\tau),
$$

and

$$
\varphi(\tau) \leq \frac{\operatorname{Vol}_{j}(\sigma)}{\operatorname{diam}^{j} \sigma} \leq c(k) \cdot \varphi(\tau),
$$

where $\varphi$ denotes the fatness of the simplex $\tau, \measuredangle(\tau, \sigma)$ denotes the (internal) dihedral angle of the face $\sigma<\tau$ and $\operatorname{Vol}_{j}(\sigma)$; diam $\sigma$ stand for the Euclidian $j$-volume and the diameter of $\sigma$, respectively. (If $\operatorname{dim} \sigma=0$, then $\operatorname{Vol}_{j}(\sigma)=1$, by convention.)

Condition A-1 is just the expression of fatness as a function of dihedral angles in all dimensions, while Condition A-2 expresses fatness as given by "large area/diameter". Diameter is important since fatness is independent of scale.

Existence of fat triangulations of Riemannian manifolds is guaranteed by the studies quoted below.

\section{A1.1 Compact manifolds:}

In the seminal contribution of Cairns, [4], the following is proved, A5[[4]] Every compact $\mathcal{C}^{2}$ Riemannian manifold admits a fat triangulation.

\section{A1.2 Open manifolds:}

A5[[36]] Every open (unbounded) $\mathcal{C}^{\infty}$ Riemannian manifold admits a fat triangulation.

\section{A1.3 Manifolds with boundary of low differentiability:}

A5[[39]] Let $M^{n}$ be an $n$-dimensional $\mathcal{C}^{1}$ Riemannian manifold with boundary, having a finite number of compact boundary components. Then, any fat triangulation of $\partial M^{n}$ can be extended to a fat triangulation of $M^{n}$.

The method used to prove Theorem A1.1, presented in [4], is to produce a point set $A \subseteq M^{n}$, that is maximal with respect to the following density condition: 


$$
d\left(a_{1}, a_{2}\right) \geq \eta, \text { for all } a_{1}, a_{2} \in A
$$

where

$$
\eta<\omega_{M} .
$$

One makes use of the fact that for a compact manifold $M^{n}$ we have $|A|<\aleph_{0}$, in order to construct the finite cell complex "cut out of $\mathrm{M}$ " by the $\nu$-dimensional Dirichlet complex, whose (closed) cells $\bar{c}_{k}=\bar{c}_{k}^{\nu}$ are given by:

$$
\bar{c}_{k}^{\nu}=\left\{x \in \mathbb{R}^{\nu} \mid d_{\text {eucl }}\left(a_{k}, x\right) \leq d_{\text {eucl }}\left(a_{i}, x\right), a_{i} \in A, a_{i} \neq a_{k}\right\},
$$

i.e. the (closed) cell complex $\left\{\bar{\gamma}_{k}^{n}\right\}$, where:

$$
\left\{\bar{\gamma}_{k}^{n}\right\}=\bar{\gamma}_{k}=\bar{c}_{k} \cap M^{n}
$$

For further details, see [4].

The proof of Theorem A1.2, [36], is based on adapting Cairns' method to the non-compact case. Essential steps in the proof are as follows.

1. Start with some compact submanifold $M_{0}^{n}$ of $M^{n}$.

2. Decompose $M^{n}$ as an exhaustion by open submanifolds, starting with $M_{0}^{n}$, namely present $M^{n}$ as,

$$
M^{n}=\bigcup_{j \in J} M_{j}^{n} ; M_{j}^{n} \subset M_{j+1}^{n} ; \overline{M_{j+1}^{n}} \backslash M_{j}^{n} \text { is compact . }
$$

3. Construct a fat triangulation of the initial compact submanifold $M_{0}^{n}$ according to [4], and iteratively extend it from $M_{j}^{n}$ to $M_{j+1}^{n}$ until a fat triangulation of the whole of $M^{n}$ is obtained.

Remark A.6 While, as already mentioned, for the full proof of Theorem A1.2 we refer the reader to the original paper [36], we should mention here that the geometric idea behind it is to delicately balance between the intrinsic curvatures of the given manifold, and the extrinsic ones obtained by its embedding in some Euclidean space (see also the brief discussion preceding Theorem 1.1 above.).

In order to prove Theorem A1.3 one first constructs two fat triangulations: $\mathcal{T}_{1}$ of a product neighbourhood $N$ of $\partial M^{n}$ in $M^{n}$ and $\mathcal{T}_{2}$ of int $M^{n}$, the existence of which follows from Peltonen's result [36], and then "mashes" the two triangulations into a new triangulation $\mathcal{T}$, while retaining their fatness. While the mashing procedure of the two triangulations is basically the one developed in the original proof of Munkres' theorem [33], the triangulation of $\mathcal{T}_{1}$ has been modified, in order to ensure the fatness of the simplices of $\mathcal{T}_{1}$. The method we have employed for fattening triangulations is the one developed in [6]. For the technical details, see [39]. 
Remark A.7 Recently, in [42], the triangulation method described above was shown to extended directly to a large class of infinite dimensional manifolds relevant to Signal and Image Processing. Moreover, a more general triangulation theorem for Hilbert cube manifolds was also shown to be relevant in Image Processing (see [42]).

\section{A2 From fat triangulations to sampling}

We first restate Theorem 1.1

Theorem ([48]): Let $\Sigma$ be a connected, non-necessarily compact smooth surface (i.e. of class $\mathcal{C}^{k}, k \geq 2$ ), with finitely many boundary components. Then, there exists a sampling scheme of $\Sigma$, with a proper density $\mathcal{D}=\mathcal{D}(p)=\mathcal{D}(\kappa)$, where $\kappa=\max \left\{\omega_{M}, \frac{1}{k(p)}\right\}$, and where $k(p)=\max \left\{\left|k_{1}\right|, \ldots,\left|k_{n}\right|\right\}$, and $\left|k_{1}\right|, \ldots,\left|k_{n}\right|$ denote the principal curvatures of $\Sigma$, at the point $p \in \Sigma$.

Proof For simplicity of the argument, and without loosing generality, let us suppose that $\kappa=\frac{1}{k(p)}$ (that is the manifold does not "turn upon itself" too fast). Then, the sampling points are provided by the vertices of the fat triangulation constructed above. ${ }^{3}$ The fact that the density is a function solely of $k=\max \left\{\left|k_{1}\right|, \ldots,\left|k_{n}\right|\right\}$ follows from Theorem A1.1 (i.e. Theorem 4.6 of [4]), and from the fact that the osculatory radius $\omega_{\gamma}(p)$ at a point $p$ of a curve $\gamma$ equals $1 / k_{\gamma}(p)$, where $k_{\gamma}(p)$ is the curvature of $\gamma$ at $p$; hence the maximal osculatory radius (of $\Sigma$ ) at $p$ is: $\omega(p)=\max \left\{\left|k_{1}\right|, \ldots,\left|k_{n}\right|\right\}=\max \left\{\frac{1}{\omega_{1}}, \ldots, \frac{1}{\omega_{n}}\right\}$. (Here $\omega_{i}, i=1, \ldots, n$ denote the minimal, respective maximal sectional osculatory radii at $p$.)

Since for unbounded surfaces it may well be that $\kappa \rightarrow \infty$, it follows that an infinite density of the sampling is possible. However, for practical implementations, where such cases are excluded, we have the following corollary:

We also quote here the following immediate corollary derived from Theorem 1.1

Corollary A.8 ([47]) Let $\Sigma, \mathcal{D}$ be as above. Assume that there exists $k_{0}>0$, such that $k_{0} \geq k(p)$, for all $p \in \Sigma$. Then, there exists a sampling of $\Sigma$ having uniformly bounded density.

In the following cases there exist $k_{0}$ as in the above Corollary A.8 ([47]):

1. $\Sigma$ is compact.

2. There exist $H_{1}, H_{2}, K_{1}, K_{2}$, such that $H_{1} \leq H(p) \leq H_{2}$ and $K_{1} \leq K(p) \leq$ $K_{2}$, for any $p \in \Sigma$, where $H, K$ denote the mean, respective Gauss curvature. (That is both mean and Gauss curvatures are pinched.)

\footnotetext{
${ }^{3}$ This happens, in fact, in the general case as well, as stressed in the previous subsection.
} 
3. The Willmore integrand $W(p)=H^{2}(p)-K(p)$ and $K$ (or $H$ ) are pinched.

Theorem 1.1 is valid for non-smooth surfaces, ([47]).

\section{A3 Reconstruction}

Definition A.9 ([33]) 1. Let $f: K \rightarrow \mathbb{R}^{n}$ be a $\mathcal{C}^{r}$ map, and let $\delta: K \rightarrow \mathbb{R}_{+}^{*}$ be a continuous function. Then $g:|K| \rightarrow \mathbb{R}^{n}$ is called a $\delta$-approximation to $f$ iff:

(i) There exists a subdivision $K^{\prime}$ of $K$ such that $g \in \mathcal{C}^{r}\left(K^{\prime}, \mathbb{R}^{n}\right)$;

(ii) $d_{\text {eucl }}(f(x), g(x))<\delta(x)$, for any $x \in|K|$;

(iii) $d_{\text {eucl }}\left(d f_{a}(x), d g_{a}(x)\right) \leq \delta(a) \cdot d_{\text {eucl }}(x, a)$, for any $a \in|K|$ and for all $x \in \overline{\operatorname{St}}\left(a, K^{\prime}\right)$.

2. Let $K^{\prime}$ be a subdivision of $K, U=\stackrel{\circ}{U}$, and let $f \in \mathcal{C}^{r}\left(K, \mathbb{R}^{n}\right), g \in$ $\mathcal{C}^{r}\left(K^{\prime}, \mathbb{R}^{n}\right) . g$ is called a $\delta$-approximation of $f$ (on $U$ ) iff conditions (ii) and (iii) above hold for any $a \in U$.

Definition A.10 ([33]) Let $f \in \mathcal{C}^{r}(K)$ and let $s$ be a simplex, $s<\sigma \in K$. Then the linear map: $L_{s}: s \rightarrow \mathbb{R}^{n}$, defined by $L_{s}(v)=f(v)$ where $v$ is a vertex of $s$, is called the secant map induced by $f$.

The motivation for having fat triangulations for manifolds in terms of reconstruction of sampled manifolds is stressed by the following theorem.

A10 [[33]] Let $f: \sigma \rightarrow \mathbb{R}^{n}$ be of class $\mathcal{C}^{k}$. Then, for $\delta>0$, there exists $\varepsilon, \varphi_{0}>0$, such that, for any $\tau<\sigma$, fulfilling the following conditions:

(i) $\operatorname{diam}(\tau)<\varepsilon$ and,

(ii) $\tau$ is $\varphi_{0}$-fat,

then, the secant map $L_{\tau}$ is a $\delta$-approximation of $f \mid \tau$.

Following Theorem A3, we use the secant map as defined in Definition A.10, in order to reproduce a PL-manifold as a $\delta$-approximation for the sampled manifold. We may now use smoothing in order to obtain a $\mathcal{C}^{\infty}$ approximation.

It should be emphasized here that Theorem 1.1 produces an infinite sequence of $P L$-approximations of the sampled manifold which, as already stated above, converge, when the diameter of simplices tends to zero, to the original sampled manifolds. Moreover, following [6], we also have that the discrete curvature measures given on the approximating manifolds converge, as measures, to the sectional curvature of the sampled manifold. This is in accordance with the fact that, in order to achieve proper reproducing of a 1-dimensional signal according to Shannon Theorem, as in [51], one has to account for an infinite summation. 